\title{
Application of magnifying narrow-band imaging endoscopy for diagnosis of early gastric cancer and precancerous lesion
}

Jing Zhang ${ }^{1,2}$, Shi-Bin Guo ${ }^{1}$ and Zhi-Jun Duan ${ }^{1 *}$

\begin{abstract}
Background: Gastric carcinoma is the second commonest cause of cancer deaths worldwide. Early detection and diagnosis of gastric cancer in the stomach is important for improving the prognosis of gastric cancer. This retrospective study was designed to investigate the value of magnifying narrow-band imaging (NBI) in the diagnosis of precancerous lesions and early gastric cancer.

Methods: This study included 122 patients who were diagnosed with early gastric cancer or precancerous gastric lesions by endoscopy. The patients underwent an examination with conventional endoscopy, magnifying NBI, and magnifying chromoendoscopy. Images resolution was evaluated, and the morphology, pit patterns and blood capillary forms of lesions were analyzed. The presence of gastric carcinoma and high grade intraepithelial neoplasia in the biopsy samples was considered as a positive pathological result, which is used to assess accuracy of endoscopic diagnosis.

Results: For image resolution, magnifying NBI and magnifying chromoendoscopy were significantly superior to magnifying conventional endoscopy in morphology, pit pattern and blood capillary form $(P<0.01)$, and magnifying NBI was significantly superior to magnifying chromoendoscopy in blood capillary form $(P<0.01)$. IV, $V_{1}$, and $\mathrm{VI}$ type of gastric pit pattern were detected in 14 cases, 43 cases, and 17 cases in patients with high grade intraepithelial neoplasia, respectively. $V_{1}$ and $\mathrm{VI}$ type of gastric pit pattern were detected in 9 cases and 39 cases in patients with early gastric cancer, respectively. The presence of irregular minute vessels and variation in the caliber of vessels was found in 109 cases. The accuracy, sensitivity, specificity, false positive rate and false negative rate for diagnosis of early gastric cancer and precancerous gastric lesions were $68.9 \%, 95.1 \%, 63.1 \%, 24.5 \%$, and 32.4\% for conventional endoscopy, 93.6\%, 92.7\%, 94.5\%, 5.7\%, and 6.9\% for magnifying NBI, and 91.3\%, 88.6\%, 93.2\%, 13.2\%, and $21.48 \%$ for magnifying chromoendoscopy, respectively.

Conclusions: This study demonstrates that magnifying NBI is superior to conventional endoscopy in the diagnosis of early gastric cancer and precancerous gastric lesions, and can be used for screening early malignancies of the stomach.
\end{abstract}

\section{Background}

Gastric carcinoma is one of the most common malignant tumors, and is the second commonest cause of cancer deaths worldwide [1]. About 870000 new gastric cancer cases are diagnosed every year on a global scale. There is a considerable geographical variation in the incidence of this cancer [2]. It is reported that incidence

\footnotetext{
* Correspondence: cathydoctor@yahoo.com

'Department of Gastroenterology, the First Affiliated Hospital of Dalian

Medical University, Dalian 0086-116011, Liaoning Province, China

Full list of author information is available at the end of the article
}

rates are high in eastern Asia, and low in Europe, North America and Africa [1]. Chinese have high gastric cancer rates with about $30 \%$ new cases diagnosed each year, which are only less than those found in Korea and Japan [3]. The prognosis of gastric carcinoma is closely related to the stage of disease at the time of diagnosis. The survival rate is greater than $90 \%$ in 5 years [4] for early gastric cancer, but the prognosis is very poor for advanced gastric cancer. Therefore, early diagnosis of gastric cancer is very important for an excellent prognosis. However, since most patients with early gastric

\section{Biomed Central}

(c) 2011 Zhang et al; licensee BioMed Central Ltd. This is an Open Access article distributed under the terms of the Creative Commons Attribution License (http://creativecommons.org/licenses/by/2.0), which permits unrestricted use, distribution, and reproduction in any medium, provided the original work is properly cited. 
carcinoma do not have specific symptoms, it is difficult to distinguish early gastric carcinoma from benign peptic ulcer or gastritis, and only about $10-20 \%$ of gastric cancers are diagnosed as early cancers in many countries [3]. With the development of technology, some endoscopic imaging modalities, such as magnifying narrow-band imaging (NBI) endoscopy, have been used recently for the diagnosis of early gastric cancer. Although many studies have reported that this endoscopic imaging modality can increase the rate of diagnosis of early cancers and precancerous lesions by enhancing visualization $[5,6]$, there are few studies about comparison between NBI and conventional endoscopy. This retrospective study was designed to compare the accuracy and sensitivity of magnifying NBI endoscopy with conventional endoscopy and chromoendoscopy for diagnosis of precancerous lesions and early gastric cancer.

\section{Methods}

\section{Patients}

This retrospective study was performed in the Department of Gastroenterology, Dalian Central hospital. During January 2008 to January 2011, a total of 14389 patients underwent endoscopic examination, and 526 lesions were detected. Among them, 122 patients were diagnosed as early gastric cancer or precancerous gastric lesions by the pathologic results, and were enrolled in this retrospective study. The ages of the 122 patients ( 83 males and 39 females) ranged from 16 to 94 years with a mean age of $63.5 \pm 14.1$ years. Early gastric cancer was defined as cancer confined to the mucosa or submucosa regardless of lymph node metastasis. In our study, precancerous lesions referred to high grade intraepithelial neoplasia, which included intraepithelial carcinoma and severe dysplasia according to the Vienna classification of gastrointestinal epithelial neoplasms [7]. All the lesions were confirmed by pathologic diagnosis of endoscopic resection or post-surgery tissue. Exclusion criteria were pre-existing or advanced gastric cancer, recent upper gastrointestinal bleeding or coagulation disorders, and severe comorbidities that may affect tolerance to upper endoscopy. The presenting manifestations were abdominal pain in 88 cases $(72.1 \%)$, distension in 52 cases (42.6\%), heartburn in 60 cases (49.2\%), belching in 28 cases (23.0\%), acid regurgitation in 32 cases $(26.2 \%)$, and nausea in 29 cases $(23.8 \%)$.

\section{Procedures}

The patients were first given careful observation in order to identify any abnormalities of the surface or the color with conventional endoscopy. After detecting lesions, magnifying chromoendoscopy and NBI magnifying endoscopy examination were followed on the same day or on another day. The morphology, pit pattern and blood capillary form of lesions were observed and recorded to determine that the lesion was malignant or benign (Figure 1). Then, biopsies were taken and studied by the same expert pathologist. The image quality was scored by two other endoscopists during the examination. Pathological diagnosis was regarded as the gold standard, and was used to assess accuracy of endoscopic diagnosis. Once lesions were diagnosed as gastric cancer or precancerous lesions, patients were underwent endoscopic resection or surgery. This study was approved by the Medical Science's ethics committee of Dalian Central hospital, patients were informed of the possible risks and benefits of participation in the study, and written informed consent was obtained from all the patients or their relatives before their examination.

\section{Instruments}

The NBI main unit was an Olympus CV-260SL and the endoscope was an Olympus GIF-H260Z (80x) (Olympus Corporation, Japan); $1.2 \%$ iodine was used for staining (Micro-Tech, Nanjing, CO., LTD).

\section{Image evaluation}

The image quality was scored as follow: 1 point: obscure; 2 points: clouding; 3 points: more clear; and 4 points: clear [8].

\section{Statistical analysis}

Data analysis was performed using SPSS 10.0 software (Chicago, IL, USA). Analysis of variance (ANOVA) or Wilcoxon statistical methods were used to determine statistical significance. All measurements in this study were expressed as mean $\pm \mathrm{SD}$. $\mathrm{P}<0.05$ was considered statistically significant.

\section{Results}

A total of 122 cases were diagnosed as early gastric cancer or precancerous lesions by pathologic diagnosis of endoscopic resection or post-surgery tissue. The lesions were located in gastric antrum in 67 cases $(54.9 \%)$, gastric angle in 29 cases (23.8\%), gastric body in 22 cases (18.0\%), and cardia and gastric fundus in 4 cases (3.3\%). The image quality of 3 modes was compared concerning the morphology, pit pattern and blood capillary form of early gastric cancer and precancerous lesions. We found that magnifying NBI and magnifying chromoendoscopy were significantly superior to magnifying conventional endoscopy in respect of morphology, pit pattern and blood capillary form $(\mathrm{P}<0.01)$, and magnifying NBI is significantly superior to magnifying chromoendoscopy in respect of blood capillary form $(\mathrm{P}<0.01)$. There is no significant difference between magnifying NBI and 


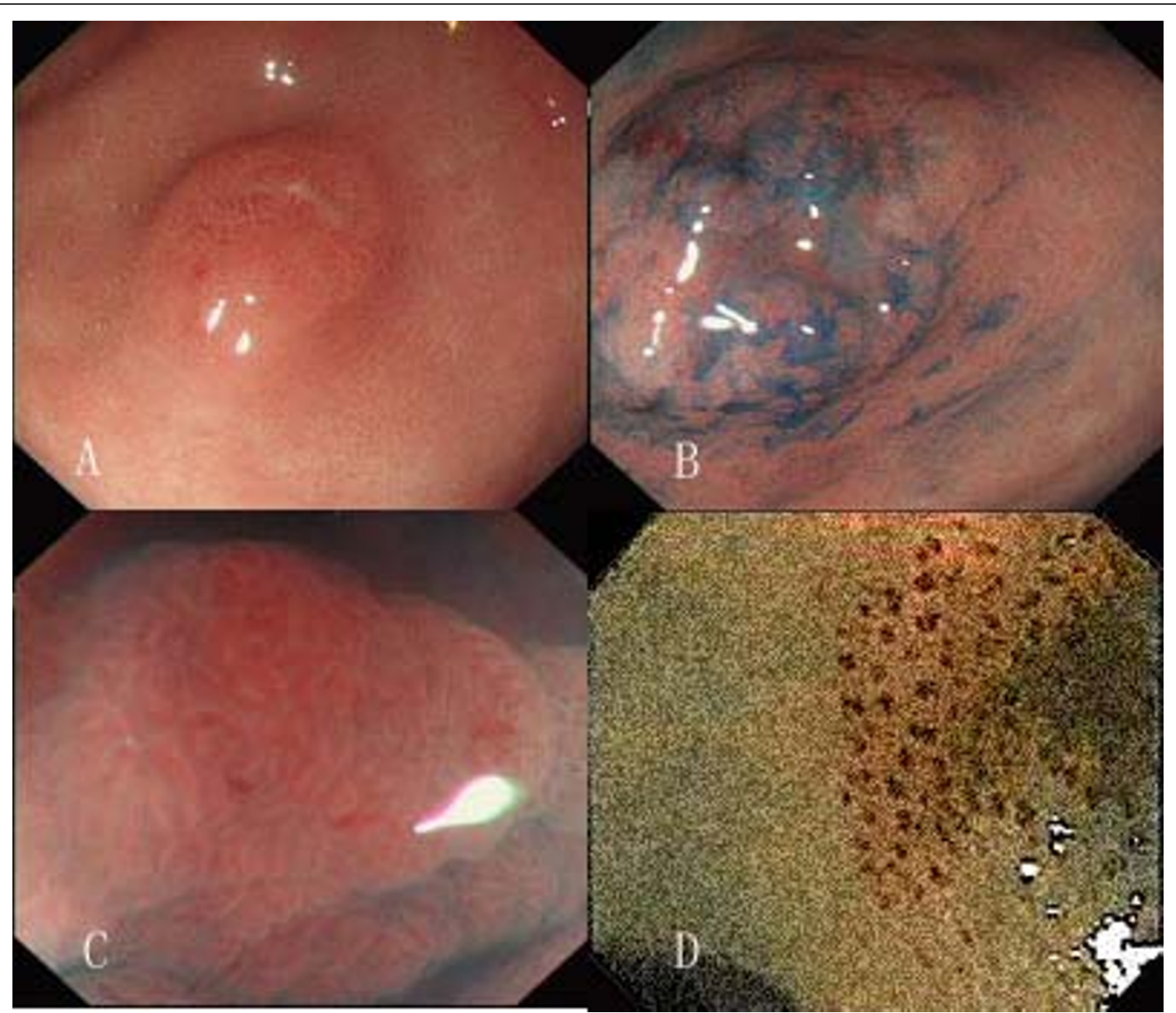

Figure 1 Demonstration of gastric precancerous lesion with conventional endoscopy(A) and iodine staining pattern (B); Pit pattern of this precancerous lesion with magnifying chromoendoscopy (C) and Blood capillary form with magnifying NBI (D).

magnifying chromoendoscopy with regard to morphology and pit pattern $(P>0.05)$ (Table 1, Figure 2).

The gastric pit pattern is classified into six types according to Sakaki [9]. In our studies, a total of 122 patients with early gastric cancer or precancerous gastric lesions were diagnosed, including 74 cases of high grade intraepithelial neoplasia and 48 cases of early gastric cancer. In patients with high grade intraepithelial neoplasia, IV type of gastric pit pattern was detected in 14 cases, $V_{1}$ type of gastric pit pattern was detected in 43 cases, and VI type of gastric pit pattern was detected in 17 cases; while in patients with early gastric cancer, $\mathrm{V}_{1}$ type of gastric pit pattern was detected in 9 cases, and VI type of gastric pit pattern was detected in 39 cases.

Table 1 Comparison of image quality among three endoscopic modalities

\begin{tabular}{|c|c|c|c|c|c|c|}
\hline \multirow[t]{2}{*}{ item } & \multirow[t]{2}{*}{ mode } & \multicolumn{4}{|c|}{ Score of image quality } & \multirow[t]{2}{*}{ total score } \\
\hline & & 1 & 2 & 3 & 4 & \\
\hline \multirow{3}{*}{ morphology } & conventional endoscopy & 26 & 33 & 63 & 0 & 281 \\
\hline & magnifying NBI & 0 & 0 & 12 & 110 & 476 \\
\hline & magnifying chromoendoscopy & 8 & 19 & 45 & 50 & 381 \\
\hline \multirow{3}{*}{ pit pattern } & conventional endoscopy & 26 & 62 & 34 & 0 & 190 \\
\hline & magnifying NBI & 0 & 0 & 25 & 97 & 463 \\
\hline & magnifying chromoendoscopy & 0 & 0 & 40 & 82 & 448 \\
\hline \multirow{3}{*}{ blood capillary form } & conventional endoscopy & 40 & 59 & 23 & 0 & 227 \\
\hline & magnifying NBI & 0 & 0 & 37 & 85 & 451 \\
\hline & magnifying chromoendoscopy & 38 & 57 & 27 & 0 & 233 \\
\hline
\end{tabular}



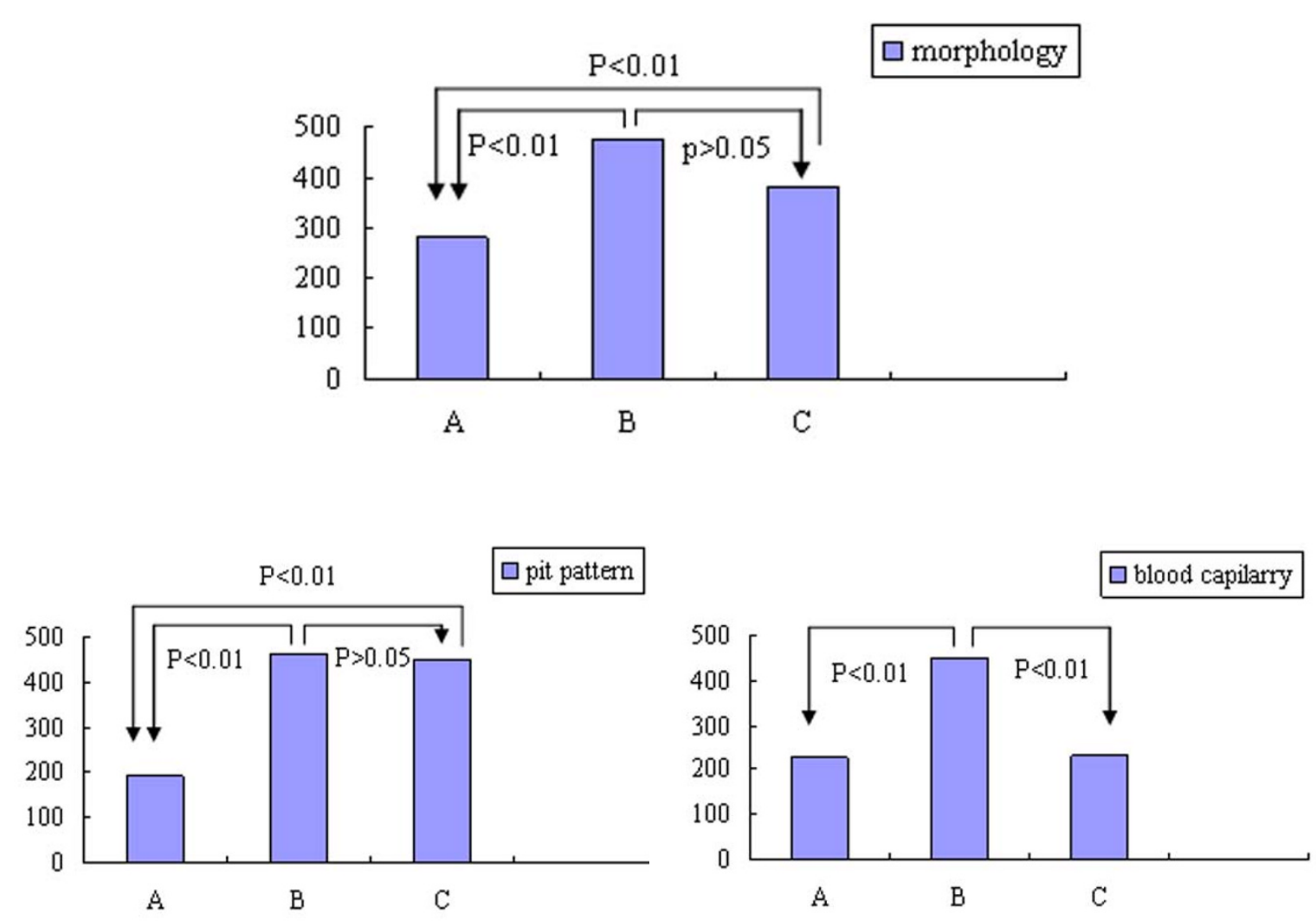

Figure 2 Comparition of image quality about morphology, pit pattern and blood capillary form among conventional endoscopy, magnifying NBI and magnifying chromoendoscopy. A represent conventional endoscopy; B represent NBI; C represent chromoendoscopy.

We also observed blood capillary form of gastric lesions. The disappearance of the normal mucosa capillary network or the presence of microvessels with irregular size, shape and distribution were found in 109 cases.

The accuracy, sensitivity, specificity, false positive rate and false negative rate of conventional endoscopic diagnosis for early gastric cancer and precancerous gastric lesions were $68.9 \%, 95.1 \%, 63.1 \%, 24.5 \%$ and $32.4 \%$, respectively, while the corresponding values of magnifying NBI were $93.6 \%, 92.7 \%, 94.5 \%, 5.7 \%$ and $6.9 \%$, respectively, and the corresponding values of magnifying chromoendoscopy were $91.3 \%, 88.6 \%, 93.2 \%, 13.2 \%$ and $21.48 \%$, respectively. Compared with conventional endoscopic, magnifying NBI was superior in the diagnosis of early gastric cancer and precancerous gastric lesions. However, there was no diagnostic difference between magnifying NBI and magnifying chromoendoscopy.

\section{Discussion}

Gastric cancer is one of the main causes of cancer death in China. Most of these patients have poor prognosis because of late presentation and diagnosis. The best tactics for dealing with gastric cancer is prevention, early detection and early treatment. It is generally believed that gastric cancer is a multi-step progression from chronic gastritis to gastric atrophy, intestinal metaplasia, dysplasia and cancer. Early gastric cancer is defined as a cancer confined to the mucosa or submucosa regardless of lymph node metastasis [10]. If gastric cancer can be detected in early stage, the prognosis for gastric cancer is excellent, the survival rate is greater than $90 \%$ in 5 years, and curative endoscopic resection may be possible in some cases with early gastric cancers and precancerous gastric lesions, without the need for surgery. Therefore, it is necessary to mass screen for symptomatic groups in high-incidence areas, and endoscopy has been considered as one of the most useful tool for detecting early gastric cancer. However, at present, only about 4$10 \%$ of patients with gastric cancer are diagnosed as early cancer in China, and the missed diagnosis of gastric cancer on endoscopy is a common occurrence. It is reported that the false-negative rates are high up to 5$19 \%[11,12]$. There may be two reasons for this situation. One reason is that the symptoms of early gastric cancer are not specific to distinguish from those of gastritis and benign peptic ulcer disease. In our group, about $90 \%$ of patients with early gastric carcinoma have heartburn, abdominal pain and discomfort in the upper 
abdomen. The other reason is that some of the lesions are so subtle that they are overlooked by inexperienced endoscopist. Without being aware of early gastric cancer, they pay much attention to the detection of gross lesions, rather than tiny changes in color, vascularity or texture, which are distinctive characteristics of early gastric cancer. There has been great advancement about the technology of endoscopic imaging in recent years, and these new technologies have improved the sensitivity in identifying early gastric cancer.

Magnifying endoscope (GIF-Q240Z) used in our study can provide magnified images up to $\mathrm{x} 80$. This new magnifying endoscope can detect minute changes of gastric mucosal surface, such as the color of lesions (same color as surrounding tissues, red or pale), the surface of lesions (flat, elevated or depressed), the presence of granules or nodules, with or without ulcer and fold change. Irregular and destruction of the minute surface pattern and color change of mucosal surface were all considered as characteristics of precancerous gastric lesion or early gastric cancer [13-15].

Dye spray chromoendoscopy can enhance the recognition of minute structural alteration caused by neoplastic changes, which is difficult to be perceived by conventional endoscopy. Magnifying chromoendoscopy is also useful in observing the surface mucosal pattern and capillary structure. By analyzing the surface structure pattern, histological changes of carcinoma, dysplasia, adenoma and hyperplasia might be suspected. Since histopathological examination of biopsy material is very important for the final diagnosis, accurate biopsy contributes to acquire the correct diagnosis of the lesion. Magnifying chromoendoscopy can improve the diagnosis of early gastric cancer and precancerous lesions in the stomach by facilitating the identification and biopsy of abnormal areas. It is difficult in some cases to identify the margins of the lesions by conventional endoscopy, especially those of superficial or flat-type lesions. Magnifying chromoendoscopy has an advantage in coping with this difficulty $[13,16]$.

Narrow-band imaging (NBI) is a new kind of endoscopic technology designed to enhance the contrast of the mucous membrane without staining. NBI uses special narrow-band filters which filter broad-band spectrum to leave a narrow-band spectrum for the diagnosis of digestive tract disease [17-19]. NBI endoscopy significantly improves diagnostic accuracy in two ways. First, because the shorter wavelength light left by the special narrow-band filter can not penetrate deeply into the mucosa, NBI improves visibility of mucosal pit pattern. Second, since the specific wavelength $415 \mathrm{~nm}$ left by special narrow-band filter corresponds to the peak absorption spectrum for hemoglobin [20], magnifying the image with NBI can give important information about microvascular pattern [21].
In our study, we find that the image quality of magnifying NBI is superior not only to magnifying conventional endoscopy in respect of morphology, pit pattern and blood capillary form of abnormal areas, but also to magnifying chromoendoscopy concerning blood capillary form. However, in our study, the image quality was scored during the examination, and the scoring system itself has subjective nature, so it would be better to score the image quality in a blinded fashion by more investigators. We also find that the diagnotic accuracy of early gastric cancer and precancerous gastric lesions by magnifying NBI is significantly higher than that of conventional endoscopy. Moreover, NBI has all the functions that conventional endoscopy has, and the NBI and conventional endoscopy pattern can be easily switched just by pushing one button [22,23].

Both NBI and chromoendoscopy can show the enhanced mucosal pattern and the microvascular structure of the mucosa by the amplificatory function[13], and mucosal pattern and microvascular structure have been regarded as distinctive characteristics of early gastric cancer and precancerous gastric lesions.

It has been known that angiogenesis is an important factor in gastrointestinal carcinogenesis [24], suggesting that the vascular pattern of gastric cancer and precancerous gastric lesion is differ from that of normal mucosa [25]. Therefore, observation of vascular pattern contributes to diagnosis of such lesions [26]. Nakayoshi [5] et al observed the microvascular networks of 165 patients with early gastric carcinoma with magnifying NBI, and found that $66.1 \%$ of differentiated adenocarcinoma had fine microvascular networks, and $85.7 \%$ of undifferentiated adenocarcinoma had corkscrew microvascular networks. Liu [27] et al also drawn the conclusion that vascular architecture was helpful in the identification of early gastric cancer. They studied the microvascular architecture with confocal endomicroscopy, and demonstrated that differentiated gastric cancerous mucosa showed hypervascularity and various caliber microvessels with irregular shapes, and undifferentiated gastric cancer showed hypovascularity and irregular short branched vessels. In our study, we found abnormal capillary patterns in 109 cases including disappearance of the normal mucosa capillary network, tortuous microvessels with irregular length and irregular arrangement, and variation in the caliber of vessels or even dilated microvessels with tortile tips. However, at present, as a new technology, there is no unified standard for diagnosis of early gastric carcinoma by using microvascular architecture.

Many studies have shown that gastric mucosa patterns of gastric cancer and precancerous gastric lesions are characteristic. Tanaka et al classified pit patterns of gastric mucosa into five types, and pointed out that 
differentiated tubular adenocarcinomas mainly showed the type IV, and poorly differentiated tubular adenocarcinomas mainly showed type V [17]. Yoshida et al distinguished gastric cancer and precancerous gastric lesions from controls by analyzing the surface structure pattern [28]. In our study, $\mathrm{V}_{1}$ and VI type of gastric pit pattern are the most common mucosa pattern of early gastric cancer and precancerous lesions. Therefore determination of pit pattern as well as microvascular architecture is very important for detecting early gastric cancer and precancerous lesion [29].

\section{Conclusions}

Magnifying NBI endoscopy has advantages in the detection of some lesions with minute changes of gastric mucosa pattern and capillary form, and facilitates the diagnosis of early gastric cancer and precancerous gastric lesions by identification and biopsy of the lesions. Clearly, it can be used for the screening of early malignancies of the stomach.

However, since the present study is uncontrolled, and the number of cases is not big enough, a further prospective, blinded study with a sufficient number of patients who have or have no gastric carcinoma is needed to assess the value of magnifying narrow-band imaging in the diagnosis of precancerous lesions and early gastric cancer in clinical practice.

\section{Author details}

${ }^{1}$ Department of Gastroenterology, the First Affiliated Hospital of Dalian Medical University, Dalian 0086-116011, Liaoning Province, China. ${ }^{2}$ This auther is currently working in Dalian Centric Hospital, Dalian 0086-116001, Liaoning Province, China.

\section{Authors' contributions}

JZ and ZJD designed the experiment, JZ and SBG performed the experiments, analyzed the data, SBG and JZ wrote the manuscript, ZJD revised it. All authors read and approved the final manuscript.

\section{Competing interests}

The authors declare that they have no competing interests.

Received: 29 August 2011 Accepted: 14 December 2011 Published: 14 December 2011

\section{References}

1. Parkin DM, Bray F, Ferlay J, Pisani P: Global cancer statistics, 2002. CA Cancer J Clin 2005, 55:74-108.

2. Sung J: Early gastric cancer: diagnosis, treatment and prevention. Eur J Gastroenterol Hepatol 2006, 18:817-819.

3. Tan YK, Fielding JW: Early diagnosis of early gastric cancer. Eur J Gastroenterol Hepatol 2006, 18:821-829.

4. Onodera H, Tokunaga A, Yoshiyuki T, Kiyama T, Kato S, Matsukura N, Masuda G, Tajiri T: Surgical outcome of 483 patients with early gastric cancer: prognosis, postoperative morbidity and mortality, and gastric remnant cancer. Hepatogastroenterology 2004, 51:82-85.

5. Nakayoshi T, Tajiri H, Matsuda K, Kaise M, Ikegami M, Sasaki H: Magnifying endoscopy combined with narrow band imaging system for early gastric cancer: correlation of vascular pattern with histopathology (including video). Endoscopy 2004, 36:1080-1084.
6. Dinis-Ribeiro M: Chromoendoscopy for early diagnosis of gastric cancer. Eur J Gastroenterol Hepatol 2006, 18:831-838.

7. The Paris endoscopic classification of superficial neoplastic lesions: esophagus, stomach, and colon: November 30 to December 1, 2002. Gastrointest Endosc 2003, 58:S3-S43.

8. Hamamoto $Y$, Endo T, Nosho K, Arimura Y, Sato M, Imai K: Usefulness of narrow-band imaging endoscopy for diagnosis of Barrett's esophagus. J Gastroenterol 2004, 39:14-20.

9. Sakaki N, lida Y, Okazaki Y, Kawamura S, Takemoto T: Magnifying endoscopic observation of the gastric mucosa, particularly in patients with atrophic gastritis. Endoscopy 1978, 10:269-274.

10. Katai H, Sano T: Early gastric cancer; concepts, diagnosis, and management. Int J Clin Oncol 2005, 10:375-383.

11. Voutilainen ME, Juhola MT: Evaluation of the diagnostic accuracy of gastroscopy to detect gastric tumours: clinicopathological features and prognosis of patients with gastric cancer missed on endoscopy. Eur J Gastroenterol Hepatol 2005, 17:1345-1349.

12. Hosokawa O, Tsuda S, Kidani E, Watanabe K, Tanigawa Y, Shirasaki S, Hayashi $H$, Hinoshita T: Diagnosis of gastric cancer up to three years after negative upper gastrointestinal endoscopy. Endoscopy 1998, 30:669-674.

13. Otsuka Y, Niwa Y, Ohmiya N, Ando N, Ohashi A, Hirooka Y, Goto H: Usefulness of magnifying endoscopy in the diagnosis of early gastric cancer. Endoscopy 2004, 36:165-169.

14. Kawamura M, Abe S, Oikawa K, Terai S, Saito M, Shibuya D, Kato K, Inomata $Y$, Ohara S, Shimosegawa T: Magnifying endoscopic findings of the surface structure of non-cancerous mucosa surrounding differentiated and undifferentiated gastric carcinoma. Dig Endosc 2011, 23:37-42.

15. Yao K, Anagnostopoulos GK, Ragunath K: Magnifying endoscopy for diagnosing and delineating early gastric cancer. Endoscopy 2009, 41:462-467.

16. Tanaka K, Toyoda H, Kadowaki S, Kosaka R, Shiraishi T, Imoto I, Shiku H, Adachi Y: Features of early gastric cancer and gastric adenoma by enhanced-magnification endoscopy. J Gastroenterol 2006, 41:332-338

17. Sharma P, Wani S, Bansal A, Hall S, Puli S, Mathur S, Rastogi A: A feasibility trial of narrow band imaging endoscopy in patients with gastroesophageal reflux disease. Gastroenterology 2007, 133:454-464.

18. Curvers W, Baak L, Kiesslich R, Van Oijen A, Rabenstein T, Ragunath K, Rey JF, Scholten P, Seitz U, Ten Kate F, Fockens P, Bergman J: Chromoendoscopy and narrow-band imaging compared with highresolution magnification endoscopy in Barrett's esophagus. Gastroenterology 2008, 134:670-679.

19. Miehlke S, Morgner A, Aust D, Madisch A, Vieth M, Baretton G: Combined use of narrow-band imaging magnification endoscopy and miniprobe confocal laser microscopy in neoplastic Barrett's esophagus. Endoscopy 2007, 39:E316.

20. Nass JP, Connolly SE: Current status of chromoendoscopy and narrow band imaging in colonoscopy. Clin Colon Rectal Surg 2010, 23:21-30.

21. Gono K, Obi T, Yamaguchi M, Ohyama N, Machida H, Sano Y, Yoshida S, Hamamoto $Y$, Endo T: Appearance of enhanced tissue features in narrow-band endoscopic imaging. J Biomed Opt 2004, 9:568-577.

22. Kara MA, Peters FP, Fockens P, ten Kate FJ, Bergman JJ: Endoscopic videoautofluorescence imaging followed by narrow band imaging for detecting early neoplasia in Barrett's esophagus. Gastrointest Endosc 2006, 64:176-185.

23. Sharma P, Bansal A, Mathur S, Wani S, Cherian R, McGregor D, Higbee A Hall S, Weston A: The utility of a novel narrow band imaging endoscopy system in patients with Barrett's esophagus. Gastrointest Endosc 2006, 64:167-175.

24. Folkman J, Klagsbrun M: Angiogenic factors. Science 1987, 235:442-447

25. Lambert R, Kuznetsov K, Rey JF: Narrow-band imaging in digestive endoscopy. ScientificWorldJournal 2007, 7:449-465.

26. Rey JF, Kuznetsov K, Lambert R: Narrow band imaging: a wide field of possibilities. Saudi J Gastroenterol 2007, 13:1-10.

27. Liu H, Li YQ, Yu T, Zhao YA, Zhang JP, Zhang JN, Guo YT, Xie XJ, Zhang TG, Desmond PV: Confocal endomicroscopy for in vivo detection of microvascular architecture in normal and malignant lesions of upper gastrointestinal tract. J Gastroenterol Hepatol 2008, 23:56-61.

28. Yoshida T, Kawachi H, Sasajima K, Shiokawa A, Kudo SE: The clinical meaning of a nonstructural pattern in early gastric cancer on magnifying endoscopy. Gastrointest Endosc 2005, 62:48-54. 
29. Mine T: The role of magnifying endoscopy in the diagnosis of early gastric carcinoma. J Gastroenterol 2006, 41:397-398.

Pre-publication history

The pre-publication history for this paper can be accessed here:

http://www.biomedcentral.com/1471-230X/11/135/prepub

doi:10.1186/1471-230X-11-135

Cite this article as: Zhang et al:: Application of magnifying narrow-band imaging endoscopy for diagnosis of early gastric cancer and precancerous lesion. BMC Gastroenterology 2011 11:135.

Submit your next manuscript to BioMed Central and take full advantage of:

- Convenient online submission

- Thorough peer review

- No space constraints or color figure charges

- Immediate publication on acceptance

- Inclusion in PubMed, CAS, Scopus and Google Scholar

- Research which is freely available for redistribution

Submit your manuscript at www.biomedcentral.com/submit 